\title{
Pediatric pulmonary hypertension
}

Pulmonary hypertension (PH) is a life-threatening disease and is characterized by pathological elevation of pulmonary pressure and associated with a heterogeneous spectrum of diseases affecting the pulmonary vasculature (1).

The care of patients with PH is a medical specialty in constant flux and shifting paradigms. There are new drugs, changing treatment strategies, renaissance of already almost forgotten surgical techniques like the Potts' shunt and last but not least improved outcome after lung transplantation as last resource for these patients.

Despite all available guidelines and recommendations, counselling remains challenging and often has to be patient tailored.

Although available treatment options have transformed the outcome of these patients, there is still a long way to go. In this series, we focus on $\mathrm{PH}$ in children. While pathobiology and clinical features share similarities in children and adults, pediatric $\mathrm{PH}$ provides many differences to adult disease, which need to be considered to provide best possible care for this challenging patient population (Figure 1).

The idea for this special series came from a very successful symposium on Pediatric Pulmonary Hypertension which took place in Münster in 2019 organized by Dr. Astrid Lammers in her role as head of the German Working Group of Pediatric Pulmonary Hypertension. Most of the invited speakers, all of them well-known experts in this field, were happy to accept our invitation to write an updated manuscript of their talks. And so, we are now proud to present this special series with the great support of the Editors of CDT.

We open with a tribute to Sheila Glennis Haworth, who sadly died in 2020. She actively contributed to our symposium in Münster by giving us the honor presenting the opening lecture on "Lung development and pathogenesis of pulmonary vascular disease". She has been a true pioneer, has participated in the last important decades with more upcoming targeted therapies and she drove clinical and basic research in the field of $\mathrm{PH}$ to strive for optimal patient care. Moreover, she has been a passionate medical professional and a true friend to many of us. Therefore, we would like to dedicate this special series to her memory (Appendix 1).

The series starts with a commentary from Lammers and Apitz on the new PH definition and its impact on pediatric $\mathrm{PH}$. According to international guidelines, $\mathrm{PH}$ used to be defined as elevation of the mean pulmonary arterial pressure (mPAP) $\geq 25 \mathrm{mmHg}$. During the 6th World Symposium on PH in 2018, a revised hemodynamic definition of PH was proposed, lowering the threshold from $\geq 25 \mathrm{mmHg}$ to $>20 \mathrm{mmHg}$ (2). This new definition has also been accepted by the Pediatric Task Force of the World Symposium to establish a uniform language and facilitate transition to adult services (3). This decision resulted in controversial debates within the expert community as the new definition may lead to overdiagnosis and overtreatment of $\mathrm{PH}$. Therefore, a critical expert discussion on the impact of the new $\mathrm{PH}$ definition in pediatric $\mathrm{PH}$ is provided (4).

While the definition of $\mathrm{PH}$ is still based on invasive hemodynamic measures, assessment of cardiopulmonary hemodynamics by cardiac catheterization and acute vasoreactivity testing (AVT) in the catheterization laboratory is critical and remains the gold standard for the diagnosis of PH. Invasive assessment of $\mathrm{PH}$ has to be performed in the context of the patient's age, medical history, and functional status. A systematic catheterization protocol is needed and has been established for pediatric $\mathrm{PH}$ by Kaestner et al. (5)

While we know that patients with vasoreactive PAH have a better prognosis, the question "how do we define vasoreactivity" is still an ongoing matter of debate. The study of Apitz et al. adds new insights into this topic and helps to understand the clinical implications of positive response during vasoreactivity testing (6). They also evaluated the clinical and prognostic value of the diastolic PA pressure (dPAP) compared to mPAP. The authors conducted a retrospective analysis of the international TOPP (Tracking Outcomes and Practice in Pediatric Pulmonary Hypertension) registry and evaluated hemodynamic data of 246 children with IPAH/HPAH. They compared the correlation between dPAP and mPAP, as well as hemodynamically-derived calculations (PAP/SAP, PVRI, TPG), using both dPAP and mPAP, at rest and during acute vasoreactivity testing (AVT) in children with idiopathic or heritable PAH. In addition, they assessed the association of these metrics (at baseline and changes after AVT) with transplant-free survival.

Mostly, the first diagnostic tool and modality in suspected PH is the transthoracic echocardiogram. Echocardiography does not only allow a comprehensive initial assessment of cardiac anatomy, dimensions and function of both ventricles, it may also confirm the suspicion of elevated pulmonary pressures and may allow for the estimation of prognosis. Lammers et al. provide 


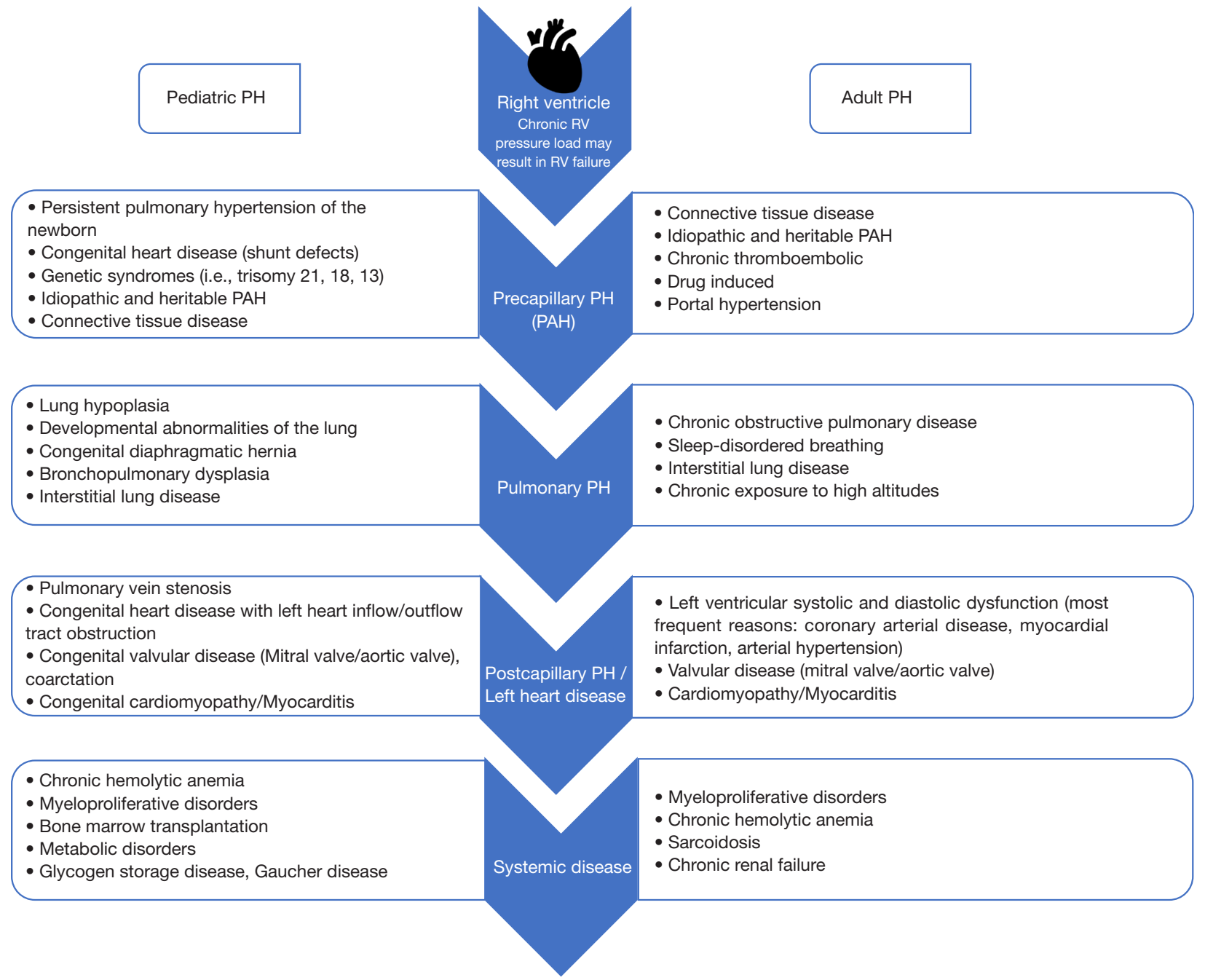

Figure 1 Similarities and differences in the etiology of pediatric and adult pulmonary hypertension.

a practical approach and a concise and clinically applicable echocardiographic guidance and present basic variables, which should be obtained at any assessment (7). Moreover, they present additional advanced echocardiographic measures, that can be applied in a research or clinical setting.

A very comprehensive assessment of the RV and the pulmonary vasculature is nowadays possible by cardiac magnetic resonance (CMR). This technique has become an essential non-invasive imaging modality in the management of $\mathrm{PH}$. Latus and Meierhofer provide many insights in its usefulness in pediatric $\mathrm{PH}$ and discuss the clinical relevance of advanced MR parameters (8).

Beyond clinical and functional assessment, as well as imaging examinations, follow-up care of patients with $\mathrm{PH}$ usually includes the measurement of biomarkers. In the meantime, many biomarkers have shown important prognostic impact in children with $\mathrm{PH}$, as excellently summarized by Ploegstra and Berger (9).

During the past few years, treatment of PAH has undergone a remarkable evolution. Due to the complex etiology and relative lack of data in children with $\mathrm{PAH}$, appropriate therapies have to be selected after careful and comprehensive review of a $\mathrm{PH}$ expert referral center according to current international recommendations. However, in pediatric PAH also specific pharmacokinetic aspects have to be considered and were presented in detail by Gorenflo and Ziesenitz (10).

Perhaps the most innovative approach in pediatric $\mathrm{PH}$ in the last decade was the renaissance of the Potts shunt as a procedure to treat children with severe PAH and systemic or suprasystemic RV pressures and imminent RV failure. Schranz 
et al. present in this series an impressive pathophysiological view on new interventional and surgical approaches to treat children with $\mathrm{PH}$ and heart failure (11).

Lung transplantation is a treatment option for selected children with end-stage $\mathrm{PH}$, which is the second most frequent indication for children requiring a lung transplantation. Sommer and Warnecke describe in detail challenges of specific considerations in children with PH regarding criteria for listing to lung transplantation, allocation policies, bridging strategies with extracorporeal support, and perioperative management (12). Bilateral lung transplantation using cardiopulmonary bypass to provide hemodynamic stability is nowadays the standard surgical approach in pediatric centers. Warnecke et al. also provide current data on long-term survival from the largest and most experienced pediatric lung transplant center in Germany.

The Eisenmenger syndrome is regarded as the most advanced form of PAH associated with congenital heart disease. There have been many insights for the treatment of Eisenmenger patients in the last decade, helping to improve outcome of these patients. Diller et al. provide a comprehensive overview on these developments and future prospects (13).

The Fontan circulation is a palliative concept for patients with univentricular hearts. The central veins are directly connected to the pulmonary arteries (cavo-pulmonary connection) to separate the pulmonary and the systemic circulation. Pulmonary blood flow is determined by central venous pressure and pulmonary vascular resistance. Therefore, systemic ventricular preload and cardiac output are dependent on a healthy lung with low pulmonary vascular resistance.

While pulmonary vascular disease and increased pulmonary resistance may result in Fontan failure, these considerations support the use of pulmonary vasodilators. The review of Becker et al. summarizes the current knowledge of the effects and efficacy of pulmonary vasodilator therapy in Fontan patients (14).

At an altitude of about 2,500 m, the barometric environmental pressure is reduced and the partial pressure of inspired oxygen drops from $21 \%$ to $15 \%$ (hypobaric hypoxia). Therefore, children with $\mathrm{PH}$ may be at risk during high-altitude exposure, by air travel or recreational activities, such as hiking and skiing. Little is known about the physiological response and risk of developing clinically relevant events on altitude exposure in pediatric PH. The review article of Herberg et al. summarizes the current clinical knowledge on moderate to high altitude exposure in patients with different forms of pediatric $\mathrm{PH}$ (15).

PAH is often associated with cardiac autonomic dysfunction, and heart rate variability (HRV) as marker of cardiac autonomic function is related to disease severity. Knowledge about the effects of physical activity on HRV is limited in PAH patients. Siaplaouras et al. assessed in their study whether HRV parameters can be influenced by a homebased exercise training program and whether respective changes are related to levels of activity (16). In their pilot study they were able to demonstrate that a workout program of 16 weeks revealed an activity level dependent effect on parameters of autonomic cardiac function in children and adolescents with $\mathrm{PAH}$. This mechanism might contribute to the positive effects of exercise training in patients with $\mathrm{PAH}$.

In this special series, we also report on rare etiologies of $\mathrm{PH}$ in children:

While in adult patients, PAH associated with connective tissue disease (CTD-PAH), including systemic sclerosis (SSc), is the most commonly identified type of disease-associated PAH, this PAH subtype is much more rarely in children. Apitz and Girschick present in their review article recent findings on diagnosis and therapy of SSc-associated PAH in children (17). Since SSc-PAH is complex and difficult to diagnose, as symptoms are non-specific and may be complicated by other diseases such as interstitial lung disease or left heart disease, regular screening for early detection of PAH is critical in pediatric SSc patients in order to improve patient outcomes.

Pulmonary veno-occlusive disease (PVOD) is a rare disease leading to $\mathrm{PH}$ and potentially death related to right heart failure and/or respiratory insufficiency. Clinical symptoms are heterogenous and nonspecific. Pfluger and Humpl provide an overview on current evidence on this rare disease (18). They state that lung transplantation is currently the only valid treatment option for patients with PVOD.

Moyamoya disease (MD) is a rare vaso-occlusive disorder that primarily affects intracranial cerebral arteries. The involvement of extracranial vessels is unusual. However, there are previous reports suggesting MD to be a systemic disorder, causing disease manifestations in vessels of other parts of the body. Krämer et al. provide a case presentation demonstrating that pulmonary arterial hypertension is a rare comorbidity in patients with $\mathrm{MD}$, especially in patients with genetic predictors such as the RNF213 mutation (19). They recommend regular echocardiographic screening for early signs of PAH in patients with MD for early detection and treatment.

By bringing together a number of specialists to refine and advance current knowledge on the topic of pediatric $\mathrm{PH}$, we hope 
that this special series may stimulate and inspire physicians and researchers in order to move forward with care of children with this progressive and complex disease, true to the motto "It's through collaboration that advances are made."

\section{Acknowledgments}

Funding: None.

\section{Footnote}

Provenance and Peer Review: This article was commissioned by the editorial office, Cardiovascular Diagnosis and Therapy for the series "Pediatric Pulmonary Hypertension". The article did not undergo external peer review.

Conflicts of Interest: Both authors have completed the ICMJE uniform disclosure form (available at http://dx.doi.org/10.21037/ cdt-21-156). The series "Pediatric Pulmonary Hypertension" was commissioned by the editorial office without any funding or sponsorship. CA and AEL served as the unpaid Guest Editors of the series. AEL reports personal fees from Actelion, outside the submitted work. The authors have no other conflicts of interest to declare.

Ethical Statement: The authors are accountable for all aspects of the work in ensuring that questions related to the accuracy or integrity of any part of the work are appropriately investigated and resolved.

Open Access Statement: This is an Open Access article distributed in accordance with the Creative Commons Attribution-NonCommercialNoDerivs 4.0 International License (CC BY-NC-ND 4.0), which permits the non-commercial replication and distribution of the article with the strict proviso that no changes or edits are made and the original work is properly cited (including links to both the formal publication through the relevant DOI and the license). See: https:/creativecommons.org/licenses/by-nc-nd/4.0/.

\section{References}

1. Berger RM, Beghetti M, Humpl T, et al. Clinical features of paediatric pulmonary hypertension: a registry study. Lancet 2012;379:537-46.

2. Simonneau G, Montani D, Celermajer DS, et al. Haemodynamic definitions and updated clinical classification of pulmonary hypertension. Eur Respir J 2019;53:1801913.

3. Rosenzweig EB, Abman SH, Adatia I, et al. Paediatric pulmonary arterial hypertension: updates on definition, classification, diagnostics and management. Eur Respir J 2019;53:1801916.

4. Lammers AE, Apitz C. Update from the World Symposium on Pulmonary Hypertension 2018: does the new hemodynamic definition of pediatric pulmonary hypertension have an impact on treatment strategies? Cardiovasc Diagn Ther 2020. doi: $10.21037 / \mathrm{cdt}-20-412$.

5. Kaestner M, Apitz C, Lammers AE. Cardiac catheterization in pediatric pulmonary hypertension: a systematic and practical approach. Cardiovasc Diagn Ther 2020. doi: 10.21037/cdt-20-395.

6. Apitz C, Berger RMF, Ivy DD, et al. Hemodynamic and prognostic impact of the diastolic pulmonary arterial pressure in children with pulmonary arterial hypertension—a registry-based analysis. Cardiovasc Diagn Ther 2021. doi: 10.21037/cdt-20-934.

7. Lammers AE, Apitz C, Michel-Behnke I, et al. A rough guide to echocardiographic assessment in children and adolescents with pulmonary hypertension. Cardiovasc Diagn Ther 2021. doi: 10.21037/cdt-21-119.

8. Latus H, Meierhofer C. Role of cardiovascular magnetic resonance in pediatric pulmonary hypertension—novel concepts and imaging biomarkers. Cardiovasc Diagn Ther 2020. doi: 10.21037/cdt-20-270.

9. Ploegstra MJ, Berger RMF. Prognostic biomarkers in pediatric pulmonary arterial hypertension. Cardiovasc Diagn Ther 2020. doi: $10.21037 / \mathrm{cdt}-20-374$.

10. Gorenflo M, Ziesenitz VC. Treatment of Pulmonary Arterial Hypertension in Children. Cardiovasc Diagn Ther 2021. doi: $10.21037 / \mathrm{cdt}-20-912$. 
11. Schranz D, Akintuerk H, Esmaeili A, et al. Heart failure therapy based on interventricular mechanics and cardio-vascular communications. Cardiovasc Diagn Ther 2020. doi: 10.21037/cdt-20-347.

12. Sommer W, Warnecke G. Lung transplantation for pediatric pulmonary arterial hypertension - quo vadis? Cardiovasc Diagn Ther 2021. doi: 10.21037/cdt-21-65.

13. Diller GP, Lammers AE, Oechslin E. Treatment of adults with Eisenmenger syndrome - state of the art in the 21 st century: A short overview. Cardiovasc Diagn Ther 2021. doi: 10.21037/cdt-21-135.

14. Becker K, Uebing A, Hansen JH. Pulmonary vascular disease in Fontan circulation-is there a rationale for pulmonary vasodilator therapies? Cardiovasc Diagn Ther 2020. doi: 10.21037/cdt-20-431.

15. Herberg U, Knies R, Müller N, Breuer J. Altitude exposure in pediatric pulmonary hypertension—are we ready for (flight) recommendations? Cardiovasc Diagn Ther 2020. doi: 10.21037/cdt-20-494.

16. Siaplaouras J, Frerix M, Apitz A, Zöller D, Apitz C. Effects of exercise training on heart rate variability in children and adolescents with pulmonary arterial hypertension: a pilot study. Cardiovasc Diagn Ther 2020. doi: 10.21037/cdt20-263.

17. Apitz C, Girschick H. Systemic sclerosisassociated pulmonary arterial hypertension in children. Cardiovasc Diagn Ther 2020. doi: $10.21037 / \mathrm{cdt}-20-901$.

18. Pfluger M, Humpl T. Pulmonary venoocclusive disease in childhood-a rare disease not to be missed. Cardiovasc Diagn Ther 2020. doi: 10.21037/cdt-20-320.

19. Krämer J, Beer M, Kaestner M, et al. Moyamoya disease associated with pediatric pulmonary hypertension—a case report. Cardiovasc Diagn Ther 2020. doi: 10.21037/cdt-20-249.

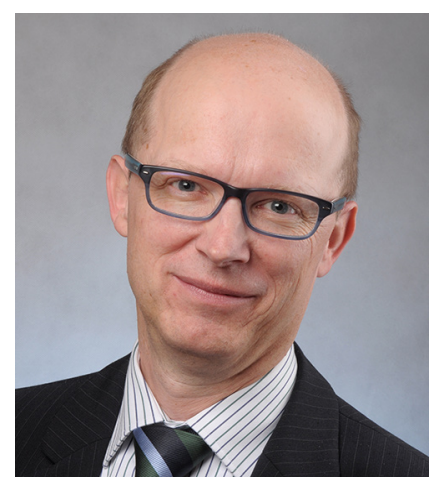

Christian Apitz

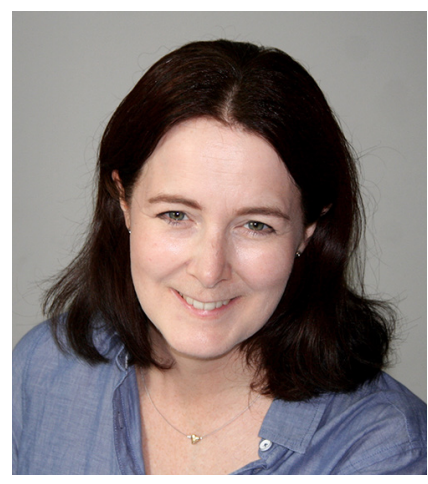

Astrid E. Lammers

Prof. Dr. Christian Apitz Division of Pediatric Cardiology, University Childrens Hospital Ulm, Ulm, Germany.

(Email: christian.apitz@uniklinik-ulm.de)

Priv.-Doz. Dr. Astrid E. Lammers, MD Department of Pediatric Cardiology, Clinic for Adult Congenital and Structural Heart Disease, University Hospital Münster, Münster, Germany. (Email: Astrid.Lammers@ukmuenster.de) Submitted Mar 12, 2021. Accepted for publication Apr 27, 2021. doi: $10.21037 / \mathrm{cdt}-21-156$ View this article at: http://dx.doi.org/10.21037/cdt-21-156

Cite this article as: Apitz C, Lammers AE. Pediatric pulmonary hypertension. Cardiovasc Diagn Ther 2021;11(4):1023-1027. doi: 10.21037/cdt-21-156 